\title{
Structure of the Universe
}

\author{
Shihao Chen \\ Department of Physics, Northeast Normal University, Changchun, China \\ Email: shchen@nenu.edu.cn,Xuan.Chen@sanofi.com,baron@american.edu,mbaron@utdallas.edu
}

How to cite this paper: Chen, S.H. (2018) Structure of the Universe. International Journal of Astronomy and Astrophysics, 8, 323-338.

https://doi.org/10.4236/ijaa.2018.84023

Received: August 8, 2018

Accepted: November 13, 2018

Published: November 16, 2018

Copyright $\odot 2018$ by author and Scientific Research Publishing Inc. This work is licensed under the Creative Commons Attribution International License (CC BY 4.0).

http://creativecommons.org/licenses/by/4.0/

\section{c) (i) Open Access}

\begin{abstract}
Based on a cosmological model without singularity, a possible structure of the universe is presented. It is proved that there must simultaneously be two sorts of symmetry breaking in the universe. The universe is composed of infinite $\mathrm{s}$-cosmic islands, infinite $\mathrm{v}$-cosmic islands and infinite transition zone. The existing and changing forms of the cosmic islands must be diverse. The cosmological principle holds only approximately within a cosmic island. No information can be exchanged between an s-cosmic island and an adjacent $\mathrm{v}$-cosmic island so that every observer thinks his cosmic island to be the whole universe. It is possible that some cosmic islands are contracting, some cosmic islands are expanding, and other cosmic islands are stable for a time. But the universe as a whole is always invariable and contains all possible existing forms of matter. To give a possible explanation for orphan quasars. To predict some characteristics of contracting large and huge black holes in a cosmic island. The characteristics of the light coming from the contracting huge black holes are that the intensity of the light is huge relatively to their distance, the red shifts are huge, the distribution of the huge red shifts and the orphan quasars are anisotropic, and luminescence spectrum is very wide.
\end{abstract}

\section{Keywords}

Cosmic Island, Quasars, Anisotropy of Huge Red Shifts, $S U(5)$ Colour Singlets, Singularity

\section{Introduction}

The Ref. [1] has presented a cosmological model without singularity based on R-W metric. According to [1], there are two sorts of matter which are called $\mathrm{s}$-matter and v-matter, respectively. The masses of the s-matter and the v-matter are all positive, but both gravitation masses are contrary to each other. S-elementary particles and v-elementary particles are perfectly symmetrical and are described by the symmetric groups $S U_{S}(5)$ and $S U_{V}(5)$, respectively. 
There are two sorts of Higgs fields which are called the s-Higgs fields and the v-Higgs fields, respectively. Both are perfectly symmetrical. and there is the coupling of s-Higgs field $\Omega_{S}$ and v-Higgs field $\Omega_{V}$. Both $\Omega_{S}$ and $\Omega_{V}$ are real fields with simple component. There are two sorts of symmetry breaking which are s-breaking and v-breaking, respectively. In the s-breaking $\left\langle\Omega_{S}\right\rangle=\langle\Omega\rangle_{0}$ and $\left\langle\Omega_{V}\right\rangle=0$, and in the v-breaking $\left\langle\Omega_{V}\right\rangle=\langle\Omega\rangle_{0}$, and $\left\langle\Omega_{S}\right\rangle=0$.

When $S U_{S}(5)$ or $S U_{V}(5)$ is not broken, i.e., $\left\langle\Omega_{S}\right\rangle=0$ or $\left\langle\Omega_{V}\right\rangle=0$, the proper masses of all s-elementary particles or all v-elementary particles are zero. Consequently, all s-elementary particles or all v-elementary particles must combine into $S U_{S}(5)$ or $S U_{V}(5)$ colour singlets. This is similar to quarks or gluons to form hadrons and mesons.

There is no singularity and there is the highest temperature $T_{\max }$ or the highest energy density $\rho_{\max }$ according to the model. The evolution of the universe has been explained [1] when the cosmological principle holds in whole universe, and the problem of the cosmological constant can be solved. Some new predictions are given [1]. A definition of the locally conservative energy-momentum tensor is given based on this model [2].

In present paper we discuss the possibility that the universe is composed of infinite cosmic islands. The cosmological principle holds approximately in a cosmic island.

This idea of multiverse or parallel universes has been proposed for a long time, such as Ref. [3]. There still are some fundamental problems of cosmology, such as singularity and cosmological constant problems, in the concept of multiverse or parallel universes. This is essentially different from the model [1].

In Section 2 it is proved that there simultaneously are two sorts of symmetry breaking in the universe; In Section 3 evolution of a huge s-black hole inside an s-cosmic island and some predictions are discussed; In Section 4 the structure of the universe is discussed; In Section 5 to give a possible explanation to the orphan quasars and some predictions; The Section 6 is the conclusions.

\section{There Simultaneously Are Two Sorts of Symmetry Breaking in the Universe}

\subsection{The Possibility That There Simultaneously Are the Two Sorts of Symmetry Breaking}

Here and below $\Omega_{S}$ and $\Omega_{V}$ are taken as an example to illustrate the change of the expectation values of Higgs fields in [1]. On the other hand, the expectation values of other Higgs fields can be determined when $\left\langle\Omega_{S}\right\rangle$ and $\left\langle\Omega_{V}\right\rangle$ are determined [1]. According to [1], there are the $S U_{S}(5)$ symmetry s-breaking or the $S U_{V}(5)$ symmetry v-breaking. It is necessary that there is the transition zone between a s-region and a v-region To consider a spherically symmetric region with its radius $r_{O}$ in a flat space. In the case,

$\mathrm{d} s=R(t) \mathrm{d} r \equiv \mathrm{d} \tilde{r}$ in the radial at a time $t$, here $R(t)$ is the scale factor in the $R W$ metric. We suppose that there is the following symmetry breaking 


$$
\begin{gathered}
\left\langle\Omega_{V}\right\rangle=\langle\Omega\rangle_{0},\left\langle\Omega_{S}\right\rangle=0, \text { when } r \geq r_{O} \\
\left\langle\Omega_{S}\right\rangle=\langle\Omega\rangle_{0},\left\langle\Omega_{V}\right\rangle=0, \text { when } r \leq r_{I}, r_{I}<r_{O}, \\
\left\langle\Omega_{S}\right\rangle=\frac{1}{2}\langle\Omega\rangle_{0}\left[\cos \tilde{k}\left(\tilde{r}-\tilde{r}_{I}\right)+1\right], \tilde{k}=k / R=\pi / R\left(r_{O}-r_{I}\right) \text {, when } r_{I}<r<r_{O}, \\
\left\langle\Omega_{V}\right\rangle=\frac{1}{2}\langle\Omega\rangle_{0}\left[-\cos \tilde{k}\left(\tilde{r}-\tilde{r}_{I}\right)+1\right], \text { when } r_{I}<r<r_{O},
\end{gathered}
$$

when

$$
T_{S}=T_{V}=0
$$

The zones where $r \leq r_{I}, r_{I}<r<r_{O}$ and $r \geq r_{O}$ are called I-zone, T-zone and O-zone, respectively. We call such a region with the boundary conditions (1)-(2) an s-cosmic island, and such a region with the boundary conditions (1)-(2) in which the subscripts $s$ and $v$ exchange, i.e., $s \rightleftarrows v$, a v-cosmic island.

Another choice of symmetry breaking easily to calculate is

$$
\begin{gathered}
\left\langle\Omega_{V}\right\rangle=\langle\Omega\rangle_{0},\left\langle\Omega_{S}\right\rangle=0, \text { when } r \geq r_{O} \\
\left\langle\Omega_{S}\right\rangle=\langle\Omega\rangle_{0},\left\langle\Omega_{V}\right\rangle=0 \text {, when } r \leq r_{I}, r_{I}<r_{O}, \\
\left\langle\Omega_{S}\right\rangle=\frac{1}{2}\langle\Omega\rangle_{0}\left[\cos \tilde{k}\left(\tilde{r}-\tilde{r}_{I}\right)+1\right], \tilde{k}=k / R=\pi / R\left(r_{O}-r_{M}\right) \text {, when } r_{I}<r<r_{M}, \\
\left\langle\Omega_{V}\right\rangle=\frac{1}{2}\langle\Omega\rangle_{0}\left[-\cos \tilde{k}\left(\tilde{r}-\tilde{r}_{I}\right)+1\right], \text { when } r_{M}<r<r_{O},
\end{gathered}
$$

when

$$
T_{S}=T_{V}=0 .
$$

We will discuss the case in another paper.

In the below discussion the coordinate $r$ and the O-zone are regarded as invariant. We discuss evolution of an s-cosmic island. It is obvious that the results are true for a $\mathrm{v}$-cosmic island as well.

It is easily seen from [1] that the expectation values of other Higgs fields and the masses of particles can be determined from (1) and (2). In the I-zone, s-elementary particles get their respective proper masses, are colourless due to $\left\langle\Omega_{S}\right\rangle=\langle\Omega\rangle_{0}$ and can form s-atoms, s-molecules and s-celestial bodies, but all v-elementary particles are massless, must possess some a sort of $S U_{V}(5)$ colours so that they must form $S U_{V}(5)$ colour singlets whose masses are not zero. There is no interaction of long range except gravitation among the $S U_{V}(5)$ colour singlets so that they can only diffusely distribute in the I-zone. Analogously, in the O-zone, v-elementary particles get their respective proper masses, are colourless due to $\left\langle\Omega_{V}\right\rangle=\langle\Omega\rangle_{0}$, and can form v-atoms, v-molecules and $\mathrm{v}$-celestial bodies, but all s-elementary particles are massless, possess some a sort of $S U_{S}(5)$ colours and must form $S U_{S}(5)$ colour singlets. There is no interaction of long range except gravitation among the $S U_{S}(5)$ colour singlets so that they can only diffusely distribute in the O-zone. In the T-zone $\left\langle\Omega_{S}\right\rangle \neq 0$ and $\left\langle\Omega_{V}\right\rangle \neq 0$, but the breaking form of the $S U_{S}(5)$ group is the same as that 
in the I-zone, and the breaking form of the $S U_{V}(5)$ group is the same as that in the O-zone. Consequently, the s-elementary particles and the v-elementary particles get all their respective proper masses. But the masses change as $\left\langle\Omega_{S}\right\rangle$ or $\left\langle\Omega_{V}\right\rangle$ continuously change. The mass $m_{S n}$ changes from $m_{S n 0}$ to 0 and the mass $m_{V n}$ changes from 0 to $m_{V n 0}$, here $m_{S n}$ and $m_{S n 0}$ are the masses of s-elementary particle $S_{n}$ in the T-zone and in the I-zone, respectively; and $m_{V n}$ and $m_{V n 0}$ are the masses of the v-elementary particle $V_{n}$ in the T-zone and in the O-zone, respectively. Consequently, the elementary particles cannot form stable bound states similar to atoms or molecules because their masses change at any moment, and there is no $S U(5)$ colour singlet, because $\left\langle\Omega_{S}\right\rangle \neq 0$ and $\left\langle\Omega_{V}\right\rangle \neq 0$. The masses of the $S U(5)$ gauge particles are not zero in the T-zone. However, it is possible that the particles form bound states similar to colour singlets for a shorter time which are called quasi colour singlets.

We compute the Higgs potential energy $V$ in the spherically symmetric T-zone. Although the calculation result is not very accurate, but $V$ to be finite is qualitatively correct. It is easily seen from [1] that when $T_{S}=T_{V}=0$,

$$
\begin{gathered}
\left\langle\Omega_{S}\right\rangle=\langle\Omega\rangle_{0}=\sqrt{\mu^{2} / \lambda} \text { and }\left\langle\Omega_{V}\right\rangle=0 \text { or }\left\langle\Omega_{V}\right\rangle=\langle\Omega\rangle_{0} \text { and }\left\langle\Omega_{S}\right\rangle=0 \\
U_{\min }=-\frac{1}{2} \mu^{2}\langle\Omega\rangle_{0}^{2}+\frac{1}{4} \lambda\langle\Omega\rangle_{0}^{4}=-\frac{\mu^{4}}{4 \lambda} .
\end{gathered}
$$

It is obvious that the Higgs potential energy exists only in the T-zone. Considering (5)-(6) and let $\bar{\Omega} \equiv\langle\Omega\rangle$, we get

$$
\begin{aligned}
V= & 4 \pi \int_{\tilde{r}_{I}}^{\tilde{r}_{0}} \mathrm{~d} \tilde{r} \cdot \tilde{r}^{2}\left\{\left[\frac{1}{2}\left(\partial_{\tilde{r}} \bar{\Omega}_{S}\right)^{2}-\frac{1}{2} \mu^{2} \bar{\Omega}_{S}^{2}+\frac{1}{4} \lambda \bar{\Omega}_{S}^{4}\right]\right. \\
& \left.+\left[\frac{1}{2}\left(\partial_{\tilde{r}} \bar{\Omega}_{V}\right)^{2}-\frac{1}{2} \mu^{2} \bar{\Omega}_{V}^{2}+\frac{1}{4} \lambda \bar{\Omega}_{V}^{4}\right]+\frac{1}{2} \Lambda \bar{\Omega}_{S}^{2} \bar{\Omega}_{V}^{2}-U_{\min }\right\} .
\end{aligned}
$$

Substituting (1) - (6) into (7), we obtain

$$
\begin{aligned}
V= & 4 \pi \int_{\tilde{r}_{I}}^{\tilde{r}_{O}} \mathrm{~d} \tilde{r} \cdot \tilde{r}^{2}\left\{\frac{\langle\Omega\rangle_{0}^{2}}{8} k^{2}(1-\cos \theta)-\frac{\langle\Omega\rangle_{0}^{2}}{8} \mu^{2}(3+\cos 2 \theta)\right. \\
& +\frac{\lambda \bar{\Omega}_{0}^{4}}{64}\left[\frac{35}{4}+7 \cos 2 \theta+\frac{1}{4} \cos 4 \theta\right] \\
& \left.+\frac{\Lambda \bar{\Omega}_{0}^{4}}{64}\left[\frac{3}{4}-\cos 2 \theta+\frac{1}{4} \cos 4 \theta\right]-U_{\min }\right\}, \theta \equiv \tilde{k}\left(\tilde{r}-\tilde{r}_{I}\right) .
\end{aligned}
$$

Substituting $\langle\Omega\rangle_{0}^{2}=\mu^{2} / \lambda$ and (6) into (7) and let $R\left(r_{O}-r_{I}\right) \equiv a$, we get

$$
\begin{gathered}
V=\frac{\pi \mu^{2}}{2 \lambda}\left\{\frac{\pi^{2}}{a}\left(R r_{I}\right)^{2}+\pi^{2}\left(R r_{I}\right)+A a+B a^{2}+C a^{3}\right\}, \\
A=\left(\frac{\pi^{2}}{3}-\frac{1}{2}\right)+\frac{3}{32} \mu^{2}\left(R r_{I}\right)^{2}\left(1+\frac{\Lambda}{\lambda}\right), \\
B=\frac{3}{32} \mu^{2}\left(R r_{I}\right)\left(1+\frac{\Lambda}{\lambda}\right),
\end{gathered}
$$




$$
C=\frac{1}{32} \mu^{2}\left[\left(1-\frac{5}{8 \pi^{2}}\right)+\left(1-\frac{15}{8 \pi^{2}}\right) \frac{\Lambda}{\lambda}\right]
$$

We regard $a$ as the variational parameter and $\tilde{r}_{I}=R r_{I}$ as invariant for a time. From

$$
\frac{\partial V}{\partial a}=0 \text { at } a=a_{0}=a_{0}\left(R r_{I}, \mu, \Lambda, \lambda\right)=a_{0}\left(\tilde{r}_{I}\right),
$$

we get the minimun $V_{\min }$ for some a definite $\tilde{r}_{I}=R r_{I}$. The pressure $F_{T}$ of the T-zone on the I-zone and the intensity of the pressure corresponding to $F_{T}$ are respectively

$$
\begin{gathered}
V_{\min }=V\left(R r_{I}, a_{0}\right), \\
F_{T}=-\frac{\partial V_{\min }}{\partial \tilde{r}_{I}}, P_{T}=\frac{F_{T}}{4 \pi \tilde{r}_{I}^{2}} .
\end{gathered}
$$

$V_{\min }\left(\tilde{r}_{I}, a_{0}\right)$ must be finite for a certain finite value of $R_{I}$. This is important, because the T-zone can exist so long as $V_{\min }\left(\tilde{r}_{I}, a_{0}\right)$ is finite, It is easily seen from (9) - (11) that

$$
a_{0} \approx \frac{\pi}{\mu}\left(\frac{32}{3(1+\Lambda / \lambda)}\right)^{1 / 2}
$$

$a_{0}$ is approximately independent of $R$ when $\quad \tilde{r}_{I}$ is large.

Taking a rough approximation of $P_{T}$ that $a_{0}$ is regarded to be independent of $\tilde{r}_{I}$, we get

$$
P_{T}=\frac{F_{T}}{4 \pi \tilde{r}_{I}^{2}}=-\frac{\mu^{2}}{4 \lambda}\left[\pi^{2}+\frac{3}{32}\left(1+\frac{\Lambda}{\lambda}\right) a_{0}^{2} \mu^{2}\right]\left[\frac{1}{a_{0} \tilde{r}_{I}}+\frac{1}{2 \tilde{r}_{I}^{2}}\right] .
$$

The energy-momentum tensor of ideal gas is

$$
T_{\mu v}=(\rho+p) u_{\mu} u_{v}-g_{\mu v} p
$$

where $\rho$ and $p$ are the gravitational mass density and the intensity of pressure of the gas. In order to consider the effect of $P_{T}$ on the evolution of the s-cosmic island, we suppose that there is a effective ideal gas with $T_{\mu \nu}$ and $u_{\mu}=u_{v}=0$ corresponding to $P_{T}$ and

$$
P_{T}=w \rho_{T}=-\rho_{T} .
$$

Consequently, the Friedmann equations become [1]

$$
\begin{gathered}
(\dot{R})^{2}+k=\eta\left(\rho_{S}-\rho_{V}-P_{T}+V_{S I}\right) R^{2}=\eta \rho_{g} R^{2} \\
\ddot{R}=-\frac{\eta}{2}\left[\left(\rho_{S}-\rho_{V}\right)+3\left(p_{S}-p_{V}\right)+2 P_{T}-2 V_{S I}\right] R=-\frac{\eta}{2}\left(\rho_{g}+3 p_{g}\right) R,
\end{gathered}
$$

where $\eta=8 \pi G / 3, k=0$ is taken, $\rho_{g}$ is the gravitational mass density, $p_{g}=\left(p_{S}-p_{V}\right)+P_{T}-V_{S I}$, and $V_{S I}$ is the s-Higgs potential energy in the I-zone. $V_{S I}=0$ when the temperature $T_{S} \approx 0$, and $V_{S I}=V_{0}$ when $T_{S} \gtrsim T_{\max }$ inside the I-zone. (16)-(17) hold only when $r<r_{I}$. Another choice different from (15) is $w \rightarrow \infty$, i.e., $\rho_{T}=0$. We will discuss it in another paper. 
Here only the effect of the intensity of pressure $P_{T}$ is considered. The reasons are as follows.

A. The potential energy $V_{\min }$ in the T-zone is the Higgs potential energy, and no gravitational potential energy coming from gravitational masses.

B. Let $V_{g T}, V_{S T}$ and $V_{V T}$ be the gravitational potential energy, s-Higgs potential energy and the v-Higgs potential energy in the T-zone, respectively, then $V_{g T} \equiv V_{S T}-V_{V T} \approx 0$ because the gravitational masses of $V_{S T}$ and $V_{V T}$ are opposite from each other and $V_{S T} \approx V_{V T}$ in the T-zone.

C. No contribution of the total Higgs potential energy $V_{\min }$ in (11) to the Einstein tensor $G_{\mu \nu}=R_{\mu \nu}-(1 / 2) g_{\mu \nu} R$ inside the I-zone, because the T-zone is spherical symmetric.

It is seen that only the effect of $P_{T}$ should be considered.

It is obvious that $V_{\min }=0$ when $R_{I} \rightarrow 0$ and $a_{0} \rightarrow 0$. This implies the T-zone will contract and it cannot exist. But, in fact, it is possible that the T-zone exists when the energy density $\rho_{V}$ of the $S U_{V}(5)$ colour singlets and the energy density $\rho_{S}$ of the s-particles in the I-zone are large enough. The reasons are as follows.

1) In the T-zone, the expectation values of the s-Higgs fields and the v-Higgs fields are different from those in the I-zone and the O-zone. But the breaking forms of these Higgs fields do not change. Hence the s-photons and v-photons in the T-zone are still massless. The s-photons are not the colour singles in the O-zone. The v-photons are not the colour singles in the I-zone as well. Consequently, when the s-photons pass the T-zone and arrive the O-zone, they cannot enter the O-zone and will be reflected back unless they and other s-particles combine to form $S U_{S}(5)$ quasi colour singlets for a shorter time. The same is true for the v-photons which arrive the I-zone from the O-zone. The s-photons reflected back to the I-zone resist the contraction of the T-zone. Analogously, the v-photons reflected back to the O-zone resist the expansion of the T-zone.

2) If a v-elementary particle $E_{V}$ enters into the T-zone from the I-zone, it will get its proper mass $m_{V E}$, and $m_{V E}$ will increase as $\left\langle\Omega_{V}\right\rangle$ continuously increases. Consequently, if a $S U_{V}(5)$ colour singlet $S_{V}$ enters into the T-zone from the I-zone, $S_{V}$ must suffer resistance coming from the T-zone due to its mass to increase. When the colour singlet $S_{V}$ enters the T-zone, $S_{V}$ is no longer a colour singlet and possibly splits into several v-elementary particles composing it, such as $E_{V}$ and $E_{V}^{C}$. The changes of $m_{V E}$ are realized by the interactions of $E_{V}$ and the v-Higgs fields via exchanging virtual Higgs particles or by the interactions of $E_{V}$ and other v-particles. The increasing of the mass $m_{V E}$ from 0 to $m_{V E 0}$ needs multiple such interactions, here $m_{V E 0}$ is the mass of $E_{V}$ when $E_{V}$ is in the O-zone. Consequently, the probability of $S_{V}$ or $E_{V}^{C}$ to pass the T-zone and to enter the O-zone must be very small. On the other hand, if $S_{V}$ gets its mass $m_{V C 0}, S_{V}$ as a whole can enter to the O-zone, here $m_{V C 0}$ is the mass of $S_{V}$ when $S_{V}$ is in the O-zone. It is obvious 
that the probability must be smaller. It is seen that when $E_{V}, E_{V}^{C}$ or $S_{V}$ passes the $\mathrm{T}$-zone and enters into the $\mathrm{O}$-zone from the I-zone, it must suffer resistance coming from the $\mathrm{T}$-zone due to the masses to increase

If $S_{V}$ does not enter the O-zone, it will return to the I-zone. If $E_{V}$ and $E_{V}^{C}$ do not enter the O-zone, they will combine into a quasi $S U_{V}(5)$ colour singlet and returns to the I-zone. The v-particles to return to the I-zone resist the contraction of the T-zone. This implies that contraction of the T-zone $\left(R_{I}\right.$ reduces) must suffer a repulsing force coming from the $V-S U_{V}(5)$ colour singlets. It is seen from the above described that when the density of the $S U_{V}(5)$ colour singlets is large enough, the contraction of the T-zone can be prevented. The v-particles entering into the $\mathrm{O}$-zone will mix with the original $\mathrm{v}$-particles in the $\mathrm{O}$-zone and cannot be distinguished. The T-zone seems to be the membrane of a balloon and the $S U_{V}(5)$ colour singlets in the I-zone seem to be gas in the balloon.

3) It is possible that a s-particle $E_{S}$ with its proper mass $m_{E S}$ enters into the T-zone from the I-zone. When it enters the T-zone, its mass $m_{E S}$ will decrease as $\left\langle\Omega_{S}\right\rangle$ continuously decreases. But $E_{S}$ is not a $S U_{S}(5)$ colour singlet, hence it cannot pass the T-zone to enter the O-zone unless $E_{S}$ and other s-particles, such as $E_{S}^{C}$, combine to form a $S U_{S}(5)$ quasi colour singlet. But the probability must be very small, because there is no interaction with long range among the s-particles except the gravitation due to $\left\langle\Omega_{S}\right\rangle \neq 0$ in the T-zone. When $E_{S}$ does not enter the O-zone, it will return back to the I-zone provided its mass renewedly increase to $m_{E S 0}, m_{E S 0}$ is the mass when $E_{S}$ is in the I-zone. The s-particles returning back to the I-zone resist the contraction of the T-zone. The $S U_{S}(5)$ quasi colour singlets can enter the O-zone. After they enter into the O-zone, they become the corresponding $S U_{S}(5)$ colour singlets, i.e., dark energy particles which cannot be observed by observers in the O-zone.

4) When $\dot{R} \neq 0, \rho_{s}$ and $\rho_{V}$ will change, hence the intensity of pressure of the I-zone on the T-zone will change.

Let $P_{S I}=p_{S}$ and $P_{V I}=p_{V}$ be the intensities of the pressure of the $\mathrm{s}$-particles and the v-colour singlets inside I-zone on the T-zone, respectively, and $\beta_{S I}$ and $\beta_{V I}$ be the probabilities of the s-particles and the $\mathrm{v}$-colour singlets inside the I-zone to penetrate the T-zone, respectively, based on the discussion above, the intensity of the pressure of the particles in the I-zone on the T-zone is

$$
P_{I}=\left(1-\beta_{S I}\right) P_{S I}+\left(1-\beta_{V I}\right) P_{V I}+\left(\zeta_{S I} \rho_{S}+\zeta_{V I} \rho_{V}\right) \dot{R}=-P_{T} .
$$

$P_{I}$ is regarded as the intensity of the counter pressure of $P_{T}$.

The discussions above are true as well for the process in which the $S U_{S}(5)$ colour singlets and the v-particles in the O-zone pass the T-zone and enter the I-zone. We do not consider the intensity of pressure of the O-zone on the T-zone for a time, because what directly affects the evolution of the I-none are $P_{T}$ and $\tilde{r}_{I}$. 


\subsection{The Relationship of the Higgs Potential Energy and Temperature}

The relationship of the Higgs potential energy and temperature is similar to that in (5.2) of [1], i.e.,

$$
\begin{aligned}
V_{e f f}^{(1) T}= & {\left[-\frac{1}{2}\left(\mu^{2}-\frac{\lambda}{4} T_{S}^{2}\right) \bar{\Omega}_{S}^{2}+\frac{\lambda}{4} \bar{\Omega}_{S}^{4}-\frac{\pi^{2}}{90} T_{S}^{4}+\frac{\mu^{2}}{24} T_{S}^{2}\right] } \\
& +\left[-\frac{1}{2}\left(\mu^{2}-\frac{\lambda}{4} T_{V}^{2}\right) \bar{\Omega}_{V}^{2}+\frac{\lambda}{4} \bar{\Omega}_{V}^{4}-\frac{\pi^{2}}{90} T_{V}^{4}+\frac{\mu^{2}}{24} T_{V}^{2}\right] \\
& +\frac{1}{2} \Lambda \bar{\Omega}_{S}^{2} \bar{\Omega}_{V}^{2}-U_{\min } .
\end{aligned}
$$

When $T_{S} \sim T_{V} \simeq T_{\max }$, inflation will occur. The inflation process is a supercooled process. After inflation, reheating process will occur. If $T_{S}<T_{V}$, s-breaking occurs; If $T_{S}>T_{V}$, v-breaking occurs.

\section{Evolution of a Cosmic Island}

Let $P_{T \max }$ be the maximum intensity of pressure of the T-zone of an s-cosmic island in contracting process. We classify s-cosmic islands into three classes. The first class is called huge cosmic islands $C_{S}^{(1)}$ whose $P_{T \max }<V_{0}$; the second class is called large cosmic islands $C_{S}^{(2)}$ whose $P_{T \max } \lesssim V_{0}$, and the third class is called small cosmic islands $C_{S}^{(3)}$ whose $P_{T \max }>V_{0}$.

Evolution of the s-cosmic islands is determined by (16)-(17) and (14).

A. Evolution of an s-cosmic island in which there is inflation process.

Let there be a huge s-cosmic island $C_{S}^{(1)}$. At $t_{-2} \rho_{g}>0, p_{g}>0, V_{S I}=0$ and $C_{S}^{(1)}$ is contracting., The temperature $T_{S}$ inside the I-zone of $C_{S}^{(1)}$ must rise due to contraction. Let $T_{S}=T_{\max }$ at $t_{-1}$. In the case, $\left\langle\Omega_{S}\right\rangle=0$ in the I-zone, the T-zone and the O-zone; $\left\langle\Omega_{V}\right\rangle=0$ in the I-zone, $\left\langle\Omega_{V}\right\rangle$ is described by (4) in the T-zone and $\left\langle\Omega_{V}\right\rangle=\langle\Omega\rangle_{0}$ in the O-zone; the masses of all elementary s-particles and v-particles inside the I-zone are zero, the s-elementary particles and the v-elementary particles can transform to each other so that $T_{V}=T_{S}=T_{\max }, V_{S I}=V_{0}, \rho_{g}=-P_{T}\left(R\left(t_{-1}\right) r_{I}\right)+V_{0}>0$, $\ddot{R} / R=-\eta\left(P_{T}\left(R\left(t_{-1}\right) r_{I}\right)-V_{0}\right)>0$, and $C_{S}^{(1)}$ continues to contract with a deceleration. The s-elementary particles will form s-colour singlets $S_{S}^{\prime} s$, and the elementary v-particles will form v-colour singlets $S_{V}^{\prime} s . S_{S}$ can freely escape out $C_{S}^{(1)}$ because of $\left\langle\Omega_{S}\right\rangle=0$ in every zone. But $S_{V}$ cannot easily escape out $C_{S}^{(1)}$ because of $\left\langle\Omega_{V}\right\rangle \neq 0$ in the T-zone. and the O-zone. Consequently, it is necessary that $\rho_{V}>\rho_{S}$ and there is such a time $t_{0}$ at which

$$
\begin{gathered}
\rho_{g}\left(t_{0}\right)=\rho_{S}\left(t_{0}\right)-\rho_{V}\left(t_{0}\right)-P_{T}\left(t_{0}\right)+V_{0}=0, \\
-\left(\rho_{g}\left(t_{0}\right)+3 p_{g}\left(t_{0}\right)\right)=-3\left[\left(p_{S}\left(t_{0}\right)-p_{V}\left(t_{0}\right)\right)+P_{T}\left(t_{0}\right)-V_{0}\right] \\
=-4\left(P_{T}\left(t_{0}\right)-V_{0}\right)>0 .
\end{gathered}
$$

This is because $\rho_{S} \sim R^{-4}, \rho_{V} \sim R^{-4}, P_{T} \sim R^{-2}, \rho_{S}<\rho_{V}, p_{S} \sim \rho_{S} / 3$ and 
$p_{V} \sim \rho_{V} / 3$ when $R$. is small. Thus, $\dot{R}=0, \ddot{R}>0$ and there must be $R>0$, i.e., there is no singularity. It is obvious that $P_{T}\left(t_{0}\right)=P_{T \max } . P_{T \max }<V_{0}$ is the condition of inflation and the physical significance of "large enough s-cosmic island". We call $P_{T}\left(t_{0}\right)=P_{T \max }$ the largest T-intensity of pressure.

It should be noted that when the symmetry $S U_{S}(5) \times S U_{V}(5)$ holds, i.e., $\left\langle\Omega_{S}\right\rangle=\left\langle\Omega_{V}\right\rangle=0$, the evolution equations are no longer those in the s-breaking, i.e., $\rho_{g}$ is no longer $\left(\rho_{S}-\rho_{V}-P_{T}+V_{0}\right)$. The evolution equations can only be determined based on the principle $(\dot{R} / R)^{2}=\eta \rho_{g} \geq 0$. As described above, $\rho_{S}<\rho_{V}$ when $\rho_{g}=0$. On the other hand, the effects of $P_{T}$ and $V_{0}$ are the same for the s-breaking and the v-breaking. Consequently, we determine the evolution equations are

$$
\begin{gathered}
(\dot{R})^{2}=\eta\left(\rho_{V}-\rho_{S}-P_{T}+V_{0}\right) R^{2}=\eta \rho_{g} R^{2}, \\
\ddot{R}=-\frac{\eta}{2}\left[\left(\rho_{V}-\rho_{S}\right)+3\left(p_{V}-p_{S}\right)+2 P_{T}-2 V_{0}\right] R
\end{gathered}
$$

It is seen from (22)-(23) that $C_{S}^{(1)}$ begin expands with a acceleration when $t>t_{0}$. There is such a time $t_{F}$ due to $C_{S}^{(1)}$ to be large enough at which $\rho_{V}\left(t_{F}\right)-\rho_{S}\left(t_{F}\right)-P_{T}\left(t_{F}\right)=0 \quad$ because $\rho_{S} \sim R^{-4}, \quad \rho_{V} \sim R^{-4} \quad$ and $\quad P_{T} \sim R^{-1}$ when $R$ increases. In the case, $\ddot{R}\left(t_{F}\right)>0$ and inflation occurs,

$$
\dot{R} / R=\sqrt{\eta V_{0}}, R(t)=R\left(t_{F}\right) \exp \sqrt{\eta V_{0}}\left(t-t_{F}\right) .
$$

The energy scale of the $S U(5)$ grant unified theory is $10^{15} \mathrm{GeV}$ and $V_{0} \sim T_{\max }^{4}=10^{60} \mathrm{GeV}^{4}$. Taking $\left(t_{H}-t_{F}\right)=10^{-33} \mathrm{~s}$, we obtain $R=10^{43} R\left(t_{F}\right)$. This is consistent with the traditional theory. After inflation, temperature $T_{S}$ and $T_{V}$ significantly drop so that $T_{S}<2 \mu / \sqrt{\lambda}$, and $T_{S}<T_{V}$ because $S_{S}$ can freely escape out $C_{S}^{(1)}$ and $S_{V}$ cannot easily escape out $C_{S}^{(1)}$ in the period $t_{H}-t_{0}$. But for $C_{S}^{(1)}$, the main factor of $T_{S}$ and $T_{V}$ to reduce is inflation, not the s-particles to escape, because the period $t_{H}-t_{0}$ is very short. This is a supercooling process.

When $t=t_{H},\left\langle\Omega_{S}\right\rangle=0 \Rightarrow\left\langle\Omega_{S}\right\rangle=\langle\Omega\rangle_{0}$ and $V_{S I}=V_{0} \Rightarrow V_{S I}=0$ occurs, but $\left\langle\Omega_{V}\right\rangle=0$ still holds. The Higgs potential energy $V_{0}$ transforms into the energies of s-particles and v-colour singlets and the energy of the T-zone. Thus, $T_{S}$ and $T_{V}$ anew rise. This is so-called reheating process. It is easily seen that after inflation, $C_{S}^{(1)}$ is still an s-cosmic island.

After the reheating process, $C_{S}^{(1)}$ will continue to expand and the evolution equations of $C_{S}^{(1)}$ is still (16)-(17) but in which $V_{S I}=0$ and $k=0$, the s-particles will form s-atoms, s-molecules and s-celestial bodies and $\rho_{S} \sim R^{-3}$ and $p_{S} \sim 0$, and the v-particles will form v-colour singlets and $\rho_{V} \sim R^{-4}$ and $p_{V} \sim \rho_{V} / 3$. Thus, it is possible that in some a period

$$
\dot{R} / R>0, \ddot{R} / R=-\frac{\eta}{2}\left[\rho_{S}-\rho_{V}-3 p_{V}+2 P_{T}\right]>0,
$$

i.e., $C_{S}^{(1)}$ will expand with an acceleration. A detailed calculation is similar to that of [1]. It it obvious that there must be such a time $t_{U}$ at which 


$$
\left(\dot{R}\left(t_{U}\right)\right)^{2}=\eta\left(\rho_{S}\left(t_{U}\right)-\rho_{V}\left(t_{U}\right)-P_{T}\left(t_{U}\right)\right) R^{2}=0,
$$

so long as $\left(t_{U}-t_{H}\right)$ is large enough, because $\rho_{S} \sim R^{-3}, \rho_{V} \sim R^{-4}$ and $P_{T} \sim R^{-1}$ when $R$ is large. It is easily seen that

$$
\ddot{R}\left(t_{U}\right)=-\frac{\eta}{2}\left[-3 p_{V}\left(t_{U}\right)+3 P_{T}\left(t_{U}\right)\right] R\left(t_{U}\right)<0 .
$$

Thus, after expansion and expansion with an acceleration, $C_{S}^{(1)}$ will stop to expand and begin to contract.

B. Evolution of an s-cosmic island in which there is no inflation process.

Let a large s-cosmic island $C_{S}^{(2)}$ with its largest T-intensity of pressure $P_{T}\left(t_{0}\right)=P_{T \max } \lesssim V_{0}$. In the case, when $T_{S}=T_{\max }$ and

$$
\begin{gathered}
\rho_{g}=\rho_{S}-\rho_{V}-P_{T}+V_{0}=0, \\
\ddot{R}=-2 \eta\left(P_{T}-V_{0}\right) R \gtrsim 0,
\end{gathered}
$$

Thus, when $t>t_{0}, C_{S}^{(2)}$ will expand.

When the temperature $T_{S}$ inside the I-zone arrives $T_{\max }$, there are two ways of $T_{S}$ to drop. The first way is $R$ increases as described above. The second way is that $R$ will slowly increase due to (28)-(29) and a lot of the s-colour singlets escape out the I-zone when $t>t_{0}$, so that $T_{S}$ drops to $T_{S O}$, here $T_{S O}$ is the temperature of the s-colour singlets outside the T-zone. Consequently, the s-breaking occurs, $\left\langle\Omega_{S}\right\rangle=0 \rightarrow\left\langle\Omega_{S}\right\rangle=\langle\Omega\rangle_{0}$, i.e., the reheating process occurs so that inflation cannot occur. In the case, it is seen from (22)-(23) that if $\rho_{g}=V_{0}$, $\ddot{R} / R=-\eta\left[2 P_{T}-V_{0}\right]<0$. Hence the condition $\rho_{g}=V_{0}$ of inflation cannot be realized when $t>t_{0}$. The evolution process after reheating process is similar to those of inflation. The main difference is that the scale factor $R$ is smaller in the reheating time in the process than that in the inflation process.

In the processes above, when $R$ increases, some energies of s-particles and $\mathrm{v}$-colour singlets transform into the Higgs potential energy in the T-zone; when $R$ decreases, a part of the energy of the Higgs potential energy in the T-zone transforms into the energies of s-particles and v-colour singlets.

It is easily seen that the I-zone of $C_{S}^{(1)}$ and the I-zone of $C_{S}^{(2)}$ are all uniform and flat. A observer outside $C_{S}^{(1)}$ or $C_{S}^{(2)}$ will detect v-particles to continuously emit out.

C. Disappearing of a small s-cosmic island

Let an s-cosmic island $C_{S}^{(3)}$ be so small that its largest T-intensity of pressure $P_{T}\left(t_{0}\right)=P_{T \text { max }}>V_{0}$, when $\rho_{g}=\rho_{S}-\rho_{V}-P_{T}+V_{0}=0$. In the case,

$$
\ddot{R}=-2 \eta\left(P_{T}-V_{0}\right) R \leq 0,
$$

$C_{S}^{(3)}$ will continue to contract, and the evolution equations are (22)-(23). The Higgs potential energy $V_{0}$ continuously transforms to the energies of s-particles and v-particles, and $\rho_{S}$ and $\rho_{V}$ continue reduce until $\rho_{S} \sim \rho_{V} \sim 0$. Finally, all Higgs potential energy of the T-zone transforms the energies of s-particles and v-particles so that the T-zone and $C_{S}^{(3)}$ disappear.

A v-observer outside $C_{S}^{(3)}$ will detect a lot of v-particles to erupt in a very 
short period. This indicates that the lifespan of a small cosmic island is very short and very small cosmic island cannot exist.

Particles inside an s-cosmic island can escape out, and some particles can enter into the s-cosmic island. But total effect is that the s-cosmic island continuously decrease its energy. Thus, when $C_{S}^{(2)}$ again contracts, it will become $C_{S}^{(3)}$ to disappear. It is seen that the lifespan of such an s-cosmic island with its $P_{T}\left(t_{0}\right)=P_{T \max } \sim V_{0}$ will be short.

\section{Evolution of a Huge S-Black Hole Inside an S-Cosmic Island and Some Predictions}

\subsection{Evolution of a Huge S-Black Hole Inside an S-Cosmic Island}

We classify s-black holes into two classes. The first class of black holes is such black holes which can contract and the temperatures inside them can arrive $T_{\max }$. The first class of black holes is divided into two sorts $B_{S}^{(2)}$ and $B_{S}^{(3)}$ which are corresponding to the two classes of s-cosmic islands $C_{S}^{(2)}$ and $C_{S}^{(3)}$. The second class of black holes is the ordinary black holes which cannot contract and the temperatures inside them cannot arrive $T_{\max }$. It is obvious that the mass and density of a first class black hole must be huger than those of a second class black hole. Evolution of a first class black hole is discussed as follows.

Let there be a spherically symmetric s-black hole $B_{S}^{(i)}, i=2$ and 3 , in the s-cosmic island $C_{S}^{(1)}$ whose total mass $M_{S}$ and density $\rho_{s}(t)$ are all large enough. There is no v-particle inside $B_{S}^{(i)}$ because its repulsion on the v-particles. Thus, $B_{S}^{(i)}$ must contract due to its gravitation and its temperature $T_{S}$ must rise. When $T_{S}$ is high enough, such as $T_{S} \geq 1 \mathrm{MeV}, \rho_{S}=g^{*} T_{S}^{4}$ and $P_{S}=\rho_{S} / 3$, the corresponding Friedmann equations are

$$
\begin{gathered}
\dot{R}^{2}+k=\eta \rho_{S} R^{2}, \ddot{R}=-(\eta / 2)\left(\rho_{S}+3 P_{S}\right) . \\
\rho_{S} R^{4}=\sigma T_{S}^{4} R^{4}=\text { const } \cdot \sigma=\left(\pi^{2} / 30\right) g^{*}, \dot{R} / R=-\dot{T}_{S} / T_{S},
\end{gathered}
$$

where $g^{*}$ is the total degrees of spin freedom. Taking $k=0$ and considering $\rho_{s}=\sigma T_{S}^{4}$, we have

$$
\begin{gathered}
\dot{R}^{2}=\eta \sigma T_{S}^{4} R^{2}, \\
\dot{T}_{S}=\sqrt{\eta \sigma} T_{S}^{3}, \frac{1}{T_{S}^{2}\left(t_{0}\right)}-\frac{1}{T_{S}^{2}\left(t_{1}\right)}=2 \sqrt{\eta \sigma}\left(t_{1}-t_{0}\right) .
\end{gathered}
$$

To consider the contraction process of $B_{S}^{(i)}$. Let $T_{S}\left(t_{0}\right)=1 \mathrm{MeV}, \sigma=25$ and $T_{S}\left(t_{1}\right)=T_{\max }=10^{15} \mathrm{GeV}$. we get $\left(t_{1}-t_{0}\right) \approx 0.3 \mathrm{~s} .10^{15} \mathrm{GeV}$ is the energy scale of the $S U(5)$ grant unified theory. At the energy scale $\left\langle\Omega_{S}\right\rangle=0$ and the expectation values of other s-Higgs fields are all zero because of the temperature effect (12), $V=V_{0}=V(0)-U_{\min }=\mu^{4} / 4 \lambda$ inside $B_{S}^{(i)}$, and $\left\langle\Omega_{S}\right\rangle=\langle\Omega\rangle_{0}$ and $V=0$ outside $B_{S}^{(i)}$. There must be a transit zone (T-zone) which satisfies (3) in the boundary zone of $B_{S}^{(i)}$. As mentioned before, $\left\langle\Omega_{V}\right\rangle=0$ in the s-cosmic island. In the case, the s-elementary particles and the v-elementary particles can transform to each other because $\left\langle\Omega_{S}\right\rangle=\left\langle\Omega_{V}\right\rangle=0$ inside $B_{S}^{(i)}$. The v-colour 
singlets can freely escape out $B_{S}^{(i)}$ due to $\left\langle\Omega_{V}\right\rangle=0$ in every zone. But the s-colour singlets cannot easily escape out $B_{S}^{(i)}$ due to $\left\langle\Omega_{S}\right\rangle=\langle\Omega\rangle_{0}$ and $V=0$ outside $B_{S}^{(i)}$. Let $\rho_{S C I}$ and $\rho_{V C I}$ be the energy densities of s-colour singlets and v-colour singlets in the I-zone, respectively, there must be $\rho_{S C I}>\rho_{V C I}$ and $T_{S I}>T_{V I}$, because the v-colour singlets can freely escape out $B_{S}^{(i)}$. Consequently, it must occur inside $B_{S}^{(i)}$ that

$$
\left\langle\Omega_{V}\right\rangle=0 \rightarrow\left\langle\Omega_{V}\right\rangle=\langle\Omega\rangle_{0}, V_{S I}=V_{0} \rightarrow V_{S I}=0,
$$

and $\left\langle\Omega_{S}\right\rangle=0$ is unchanged. Thus, a v-cosmic island called $C_{V}^{(i)}$ appears. It is seen from (35) that a huge s-black hole $B_{S}^{(i)}$ can transform into a v-cosmic island $C_{V}^{(i)}$ inside an s-cosmic island and it is necessary that there simultaneously are the two sorts of symmetry breaking in the universe so long as there are huge black holes inside $C_{S}^{(1)}$.

Evolution of $C_{V}$ can be determined by the discussions similar to those in last subsection of above section, so long as to exchange the subscripts $S$ and $V$ in the subsection. The v-cosmic islands $C_{V}^{(2)}$ and $C_{V}^{(3)}$ are corresponding to $C_{S}^{(2)}$ and $C_{S}^{(3)}$, respectively.

The inflation or expansion process after contraction of an s-cosmic island or a $\mathrm{v}$-cosmic island in the universe is similar to the following process. To suppose that there is an infinite planar rubber membrane, its a zone of radius $R$ becomes semi-spherical due to being heated. There is no significant change of its border, but the area of this zone has significantly increased in the process. Then the semi-spherical gradually become flat and tis border significantly increases.

It is obvious that the evolution of the first class of black hole is essentially different from that of the second class of black holes. It is possible that a second class black hole will disappear by the Hawking radiation.

It is seen from the above mentioned that s-matter and v-matter can transform into each other when the temperature $T$ inside a black hole or a cosmic island arrives the highest temperature $T_{\max }$.

\subsection{The Characteristics of the V-Cosmic Island and Some Predictions}

1) The v-cosmic island inside a the s-cosmic island $C_{S}^{(1)}$ cannot be very large and its largest T-intensity of pressure $P_{T}\left(t_{0}\right)=P_{T \max } \sim V_{0}$, because it stems from a huge black hole inside $C_{S}^{(1)}$. Hence there are the actual v-cosmic islands to be the two classes $C_{V}^{(2)}$ and $C_{V}^{(3)} \cdot C_{V}^{(2)}$ and $C_{V}^{(3)}$ are similar to $C_{S}^{(2)}$ and $C_{S}^{(3)}$, their lifespan are all short.

2) Huge black holes can only be formed in earlier times of the s-cosmic island $C_{S}^{(1)}$, because $C_{S}^{(1)}$ expands and the celestial bodies inside $C_{S}^{(1)}$ are away from each other so that the celestial bodies cannot gather to form a huge black late. Consequently, the huge black holes can only appear in earlier times.

3) There is no s-celestial body around $C_{V}^{(i)}$ because the repulsion $C_{V}^{(i)}$ on s-celestial bodies. After the s-black hole $B_{S}^{(i)}$ transforms into the v-cosmic island $C_{V}^{(i)}$, the celestial bodies around $B_{S}^{(i)}$ must go away. The v-cosmic island 
$C_{V}^{(i)}$ must be solitary inside the s-cosmic island.

4) $C_{V}^{(2)}$ and $C_{V}^{(3)}$ can emit a lot of s-particles and v-colour singlets. Particularly, when $C_{V}^{(2)}$ and $C_{V}^{(3)}$ will disappear, they will emit huge quantity of s-particles and v-colour singlets. A observer in the earth cannot detect the v-colour singlets, but can detect the s-particles. Only s-photons and neutrinos can be detected. Other s-particles are hard detected because they must suffer many interactions on their long roads or have decayed.

5) The characteristics of the detected s-photons and s-neutrinos on the earth as follows.

a) The red shifts of the detected s-photons and the s-neutrinos must be very large, because $C_{v}^{(2)}$ and $C_{v}^{(3)}$ must be formed in earlier times and their lifespan are all short.

b) The intensities of the photons and the neutrinos are huge relatively to their distance, and the luminescence spectrum is very wide..

c) The headstreams of the detected s-photons and s-neutrinos must be solitary due to the repulsion of $C_{v}^{(2)}$ and $C_{v}^{(3)}$ on s-celestial bodies.

Based the characteristics, we guess that

a) $C_{v}^{(2)}$ and $C_{v}^{(3)}$ are so-called orphan quasars;

b) Distribution of the orphan quasars is anisotropic, because it is possible that the earth is not in the centre of the present s-cosmic island $C_{S}^{(1)}$.

\section{Structure of the Universe}

\subsection{The Universe Is Composed of Infinite S-Cosmic Islands, V-Cosmic Islands and Transition Regions}

As described above, the s-cosmic islands and the $\mathrm{v}$-cosmic islands can simultaneously exist. Based on this, we think that the universe is composed of infinite s-cosmic islands, infinite $\mathrm{v}$-cosmic islands and infinite transition regions among the s-cosmic islands and the v-cosmic islands. It is necessary that the cosmic islands adjacent to an s-cosmic island must be v-cosmic islands, because if two s-cosmic islands are adjacent, both will combine into a larger s-cosmic island. This is true for two adjacent v-cosmic islands as well. No information can be exchanged between an s-cosmic island and a adjacent v-cosmic island so that every observer thinks his cosmic island to be the whole universe. The evolution process of a huge cosmic island is as follows: Contraction, contraction stops, expansion, inflation, reheating process, expansion, expansion with an acceleration, expansion with a deceleration, expansion stops and contraction begin. There is no inflation process in the evolution process of a larger and small cosmic island. Every cosmic island has its life and death. The existing and changing forms of the cosmic islands must be diverse. It is possible that some cosmic islands are contracting, some cosmic islands are expanding, and other cosmic islands are stable for a time. Some cosmic islands are forming, and some cosmic islands are disappearing, some cosmic islands are combining to form a larger cosmic island, and some cosmic islands are splitting into few smaller 
cosmic island. Every existence has its birth and death, and is changing. Only the universe as a whole is infinite and always unchanging, and contains all possible existing and changing forms.

The interior of cosmic island is uniform, but is not absolutely isotropic for such an observer who is not in the centre of his cosmic island. Thus, the cosmological principle holds only approximately within a cosmic island because every cosmic island must be finite. There is no singularity in every cosmic island and in the whole universe.

There are many s-black holes inside a huge s-cosmic island. A huge s-black hole can transform into a v-cosmic island in the huge s-cosmic island. The evolution of the v-cosmic island is the same as that of a large or small s-cosmic island. An observer in the s-cosmic island can detect the s-photons and neutrinos emitted by the v-cosmic island.

The present cosmological model is essentially different from the conventional cosmological theory. If the universe comes from a singularity as the conventional theory, there must be only one sort of breaking, there is no cosmic island, and the universe as a whole is variational.

\subsection{No Information Can Be Exchanged between an S-Cosmic Island and an Adjacent V-Cosmic Island}

When $\left\langle\Omega_{S}\right\rangle=\langle\Omega\rangle_{0}$ and $\left\langle\Omega_{V}\right\rangle=0$ or $\left\langle\Omega_{V}\right\rangle=\langle\Omega\rangle_{0}$ and $\left\langle\Omega_{S}\right\rangle=0$, the masses of the Higgs particles $\Omega_{V}$ and $\Omega_{S}$ are all very large [1]. Hence the interactions between s-particles and v-colour singlets by exchanging the Higgs particles must be very weak so that they may be ignored. The repulsion between s-particles and v-colour singlets are very weak and may be ignored as well. Consequently, it is impossible that v-colour singlets are detected in an s-cosmic island, It is true as well that s-colour singlets cannot be detected in a v-cosmic island. Colour singlets have only the cosmological effects and are regarded as dark energy [1].

Let a $S U_{V}(5)$ colour singlet $S_{V}$ in an s-cosmic island enter into an adjacent v-cosmic island. $S_{V}$ is a dark energy particle in the s-cosmic island so that $S_{V}$ cannot carry any information to the v-cosmic island. After $S_{V}$ enters the v-cosmic island, it will splits into few v-particles whose proper masses are not zero. The v-particles and the original v-particles in the v-cosmic island mix together and cannot be differentiate from each other.

Let s-particles in the s-cosmic island enter into the T-zone, and some s-particles combine each other to form a quasi $S U_{S}(5)$ colour singlet $\tilde{S}_{S}$ in the T-zone, then $\tilde{S}_{S}$ can enter into the v-cosmic island and become $S U_{S}(5)$ colour singlet $S_{S} . S_{S}$ cannot be detected by v-observers because there is no interaction between $S_{S}$ and v-particles. Such s-particles which do not combine to quasi s-colour singlets in the T-zone cannot enter the v-cosmic island. Consequently, the s-cosmic island cannot convey any information to the s-cosmic island, and vice verse. Thus, the observers in every cosmic island will 
all think their cosmic island to be the whole universe.

Although no information can be exchange between our cosmic island and the adjacent $\mathrm{v}$-cosmic islands, some s-colour singlets inside the $\mathrm{v}$-cosmic islands can enter into our cosmic island. The s-colour singlets entering into our cosmic island are visible particles. Consequently, in fact, the boundary of our cosmic island is slightly anisotropic, because the distribution of the adjacent v-cosmic islands. Around our s-cosmic island is not symmetric. Of course, the slight anisotropy is too hard observed.

\section{Conclusions}

Based on [1], a possible structure of the universe is presented. It is proved that there must simultaneously be two sorts of symmetry breaking in the universe. The universe is composed of infinite s-cosmic islands, infinite v-cosmic islands and infinite transition zone. It is necessary that the cosmic islands adjacent to an s-cosmic island must be $\mathrm{v}$-cosmic islands. No information can be exchanged between an s-cosmic island and an adjacent v-cosmic island so that every observer thinks his cosmic island to be the whole universe.

The evolution process of a huge cosmic island is as follows: contraction, contraction stops, expansion, inflation, reheating process, expansion, expansion with an acceleration, expansion with a deceleration, expansion stops and contraction begin. There is no inflation process in the evolution process of a larger and small cosmic island.

The existing and changing forms of the cosmic islands must be diverse. It is possible that some cosmic islands are contracting, some cosmic islands are expanding, and other cosmic islands are stable for a time. But the universe as a whole is infinite, always invariable and contains all possible existing and changing forms of matter.

The cosmological principle holds only approximately within a cosmic island. To give a possible explaintion for orphan quasars. To predict some characteristics of contracting large and huge black holes in a cosmic island. The characteristics of the light coming from the contracting huge black holes are that the intensity of the light is huge relatively to their distance, the red shifts are huge, the distribution of the huge red shifts and the orphan quasars is anisotropic, and luminescence spectrum is very wide.

\section{Acknowledgements}

This work is supported by National Natural Science Foundation of China (No. 11075064). I am very grateful to them.

\section{Conflicts of Interest}

The authors declare no conflicts of interest regarding the publication of this paper.

\section{References}

[1] Chen, S.H. (2014) A Cosmological Model without Singularity Based on RW Metric 
(1). International Journal of Astronomy and Astrophysics, 4, 264-293. https://doi.org/10.4236/ijaa.2014.41023

[2] Chen, S.H. (2016) A Locally Conservative Energy-Momentum Tensor in the General Relativity Based on a Cosmological Model without Singularity. Journal of Modern Physics, 7, 4. https://doi.org/10.4236/jmp.2016.73027

[3] Hubbard, K.A. (ed.) (2008) The In Ationary Multiverse. Frontier Science, 2, 37-44. 